\title{
Some Criteria for the Asymptotic Behavior of a Certain Second Order Nonlinear Perturbed Differential Equation
}

\author{
Aydin Tiryaki \\ Department of Mathematics and Computer Science, Izmir University, Izmir, Turkey \\ Email: aydin.tiryak@izmir.edu.ti
}

Received March 13, 2012; revised April 26, 2012; accepted May 6, 2012

\begin{abstract}
In this paper we give sufficient conditions so that for every nonoscillatory $u(t)$ solution of $\left(r(t) \psi(u) u^{\prime}\right)^{\prime}+Q(t, u)=P\left(t, u, u^{\prime}\right)$, we have $\liminf _{t \rightarrow \infty}|u(t)|=0$. Our results contain the some known results in the literature as particular cases.
\end{abstract}

Keywords: Perturbed Differential Equations; Nonoscillatory Solution; Asymptotic Behavior

\section{Introduction}

This paper is concerned with the problem of asymptotic behavior of the second order nonlinear perturbed differential equation

$$
\left(r(t) \psi(u) u^{\prime}\right)^{\prime}+Q(t, u)=P\left(t, u, u^{\prime}\right)
$$

where

$$
r \in C\left(I, R^{+}\right), \psi \in C\left(R, R^{+}\right), R=(-\infty, \infty), I=\left[t_{0}, \infty\right) .
$$

Throughout the paper according to the results we shall impose the following conditions:

$\left(\mathrm{H}_{1}\right)$ Let $f \in C^{1}(R, R)$ and there exists a constant $k>0$ such that $\frac{f^{\prime}(x)}{\psi(x)} \geq k$ and $f(x) x>0$ for $x \neq 0$,

$\left(\mathrm{H}_{2}\right) \quad Q \in C(I \times R, R)$ and there exists a continuous function $q(t)$ such that $\frac{Q(t, x)}{f(x)} \geq q(t)$ for $x \neq 0$,

$\left(\mathrm{H}_{3}\right) \quad P \in C(I \times R \times R, R)$ and there exists a continuous function $p(t)$ such that $\frac{P(t, x, y)}{f(x)} \leq p(t)$ for $x \neq 0, y \neq 0$

$\left(\mathrm{H}_{4}\right) \quad P \in C(I \times R \times R, R)$ and there exists a continuous function $p(t)$ such that $\frac{P(t, x, y)}{f(x)} \leq \frac{p(t) y}{f(x)}$ for $x \neq 0, y \neq 0$

$\left(\mathrm{H}_{5}\right) \int_{\varepsilon}^{\infty}\left(\frac{\psi(s)}{f(s)}\right) \mathrm{d} s<\infty$ and $\int_{-\varepsilon}^{-\infty}\left(\frac{\psi(s)}{f(s)}\right) \mathrm{d} s<\infty$ for every $\varepsilon>0$.

We shall also restrict our attention only to the solution of the differential Equation (1.1) which exist on some ray of the form $\left[t_{0}, \infty\right)$.

The oscillatory behavior of the solution of second order ordinary differential equations including the existence of oscillatory and nonoscillatory solutions has been the subject of intensive investigation. This problem has received the attention of many others. See for example, [1-5]. Since the publication of Hammet's paper in [6] in 1971, the asymptotic behavior of the solution of the ordinary and functional differential equations has been widely discussed in the literature [2,4,7-9].

In this paper we give sufficient conditions so that for every nonoscillatory $u(t)$ solution of (1), we have $\liminf _{t \rightarrow \infty}|u(t)|=0$. Our results contain the results in [10] as particular cases.

\section{Main Results}

In this section we prove our main results.

Theorem 2.1. Let conditions $\left(\mathrm{H}_{1}\right),\left(\mathrm{H}_{2}\right),\left(\mathrm{H}_{3}\right)$ and $\left(\mathrm{H}_{5}\right)$ hold. If there exists a differentiable function $\rho: I \rightarrow R^{+}$ such that

$$
\begin{gathered}
\rho^{\prime}(t) \geq 0, \\
\int_{\infty}^{\infty} \frac{\mathrm{d} t}{\rho(t) r(t)}<\infty
\end{gathered}
$$

and

$$
\lim _{t \rightarrow \infty} \int_{t_{0}}^{t} \frac{1}{\rho(s) r(s)}\left(\int_{t_{0}}^{s}[A(\tau)] \mathrm{d} \tau\right) \mathrm{d} s=\infty
$$


where

$$
A(t)=\rho(t)\left[q(t)-p(t)-\frac{v(t)}{4 k}\left(\frac{\rho^{\prime}(t)}{\rho(t)}\right)^{2}\right] .
$$

Then for every nonoscillatory solution $u$ of Equation (1), we have $\liminf _{t \rightarrow \infty}|u(t)|=0$.

Proof. Let $u(t)$ be a nonoscillatory solution of (1). We may assume that $u(t) \neq 0$ for $t \geq t_{1} \geq t_{0}$. Define

$$
w(t)=\rho(t) \frac{r(t) \psi(u(t)) u^{\prime}(t)}{f(u(t))} .
$$

Differentiating (5) and making use of (1) and from hypothesis $\left(\mathrm{H}_{1}\right),\left(\mathrm{H}_{2}\right)$ and $\left(\mathrm{H}_{3}\right)$, it follows that

$$
w^{\prime}(t) \leq \frac{\rho^{\prime}(t)}{\rho(t)}|w(t)|-p(t)(q(t)-p(t))-k \frac{|w(t)|^{2}}{p(t) r(t)}
$$

By using the inequality

$$
D X-E X^{2} \leq \frac{1}{4} D^{2} E^{-1}, D \geq 0, E>0, X \geq 0
$$

we get

$$
w^{\prime}(t) \leq-\rho(t)\left[q(t)-p(t)-\frac{1}{4 k} r(t)\left(\frac{\rho^{\prime}(t)}{\rho(t)}\right)^{2}\right]
$$

Integrating this inequality, from $t_{1}$ to $t$, we get

$$
\rho(t) \frac{r(t) \psi(u(t)) u^{\prime}(t)}{f(u(t))} \leq w\left(t_{1}\right)-\int_{t_{1}}^{t} A(s) \mathrm{d} s, t \geq t_{1} \geq t_{0}
$$

Dividing (9) by $\rho(t) r(t)$ and hence integrating from $t_{1}$ to $t$ we obtain

$$
\begin{aligned}
& \int_{u\left(t_{1}\right)}^{u(t)} \frac{\psi(s)}{f(s)} \mathrm{d} s \\
& \leq w\left(t_{1}\right) \int_{t_{1}}^{t} \frac{\mathrm{d} s}{\rho(s) r(s)}-\int_{t_{1}}^{t} \frac{\mathrm{d} s}{\rho(s) r(s)}\left(\int_{t_{1}}^{s} A(\tau) \mathrm{d} \tau\right) \mathrm{d} s
\end{aligned}
$$

Using (4) we get

$$
\liminf _{t \rightarrow \infty} \int_{u\left(t_{1}\right)}^{u(t)} \frac{\psi(s)}{f(s)} \mathrm{d} s=-\infty
$$

If $\liminf _{t \rightarrow \infty} u(t)>0$, then there exists a positive constant $c$ such that $u(t) \geq c$ for all $t \geq t_{1}$ and consequently, by $\left(\mathrm{H}_{5}\right)$

$$
\left|\int_{u\left(t_{1}\right)}^{u(t)} \frac{\psi(s)}{f(s)} \mathrm{d} s\right| \leq \int_{c}^{\infty} \frac{\psi(s)}{f(s)} \mathrm{d} s<\infty
$$

which contradicts (11). Thus we must have $\lim _{t \rightarrow \infty} \inf u(t)=0$. The proof for the case $\liminf _{t \rightarrow \infty} u(t)<0$ for $t \geq t_{1}$ is similar and hence is omitted.

We note that when $\rho(t) \equiv 1,\left(\mathrm{H}_{1}\right)$ condition can be weakened. Indeed from the proof of Theorem 2.1, the following result can obtain easily.

Theorem 2.2. Let conditions $\left(\mathrm{H}_{1}\right),\left(\mathrm{H}_{2}\right),\left(\mathrm{H}_{3}\right)$ and $\left(\mathrm{H}_{5}\right)$ hold. Suppose that

$$
\int^{\infty} \frac{\mathrm{d} t}{r(t)}<\infty, \psi(x)>0 \text { and } \frac{f^{\prime}(x)}{\psi(x)} \geq 0 \text { for } x \neq 0,
$$

and

$$
\limsup _{t \rightarrow \infty} \int_{t_{0}}^{t} \frac{1}{r(s)}\left(\int_{t_{0}}^{s}(q(\tau)-p(\tau)) \mathrm{d} \tau\right) \mathrm{d} s=\infty .
$$

Then for every solution $u(t)$ of Equation (1), we have $\liminf _{t \rightarrow \infty} u(t)=0$.

By taking $\left(\mathrm{H}_{4}\right)$ instead of $\left(\mathrm{H}_{3}\right)$ we obtain the following result which can be applied for example to the damped equation

$$
\left(r(t) \psi(u) u^{\prime}\right)^{\prime}+b(t) u^{\prime}+q(t) f(u)=0, t \geq t_{1} \geq t_{0} .
$$

Theorem 2.3. Let conditions $\left(\mathrm{H}_{1}\right),\left(\mathrm{H}_{2}\right),\left(\mathrm{H}_{4}\right)$ and $\left(\mathrm{H}_{5}\right)$ hold. Suppose that

$$
\psi(x) \geq c>0 .
$$

If there exists a differentiable function $\rho: I \rightarrow R^{+}$ such that (3) holds and,

$$
\limsup _{t \rightarrow \infty} \int_{t_{0}}^{t} \frac{1}{\rho(s) r(s)}\left(\int_{t_{0}}^{s} B(\tau) \mathrm{d} \tau\right) \mathrm{d} s=\infty
$$

where

$$
B(t)=\rho(t)\left[q(t)-\frac{r(t)}{4 k}\left(\frac{\left|\rho^{\prime}(t)\right|}{\rho(t)}+\frac{|p(t)|}{c r(t)}\right)^{2}\right]
$$

then for every solution $u(t)$ of Equation (1), we have $\liminf _{t \rightarrow \infty} u(t)=0$.

Proof. Let $u(t)$ be a nonoscillatory solution of Equation (1). We may assume that $u(t) \neq 0$ for $t \geq t_{1} \geq t_{0}$. Differentiating (5) and making use of (1) and from hypothesis $\left(\mathrm{H}_{1}\right),\left(\mathrm{H}_{2}\right),\left(\mathrm{H}_{4}\right)$ and $(12)$, as in the proof of Theorem 2.1, we can obtain easily that

$$
w^{\prime}(t) \leq-\rho(t)\left[q(t)-\frac{r(t)}{4 k}\left(\frac{\left|\rho^{\prime}(t)\right|}{\rho(t)}+\frac{|p(t)|}{c r(t)}\right)^{2}\right]
$$

Integrating this inequality from $t_{1}$ to $t$ we get

$$
\rho(t) \frac{r(t) \psi(u(t)) u^{\prime}(t)}{f(u(t))} \leq w\left(t_{1}\right)-\int_{t_{1}}^{t} B(s) \mathrm{d} s
$$


Dividing (15) by $\rho(t) r(t)_{1}$ and hence integrating from $t_{1}$ to $t$ we obtain

$$
\liminf _{t \rightarrow \infty} \int_{x\left(t_{1}\right)}^{x(t)} \frac{\psi(u)}{f(u)} \mathrm{d} u=-\infty
$$

The rest of of the proof is similar to that of Theorem 2.1 and hence is omitted.

Remark 2.1. Grace and Lalli, consider the following equation

$$
\left(a(t) \psi(x(t)) x^{\prime}(t)\right)^{\prime}+p(t) x^{\prime}(t)+q(t) f(x(t))=0
$$

in [10] and give a similar result. But if we compare Theorem 2.3 with Theorem 2 in [10], we observe that they have a condition such as $\rho^{\prime}(t) p(t) \leq 0$ for $t \geq t_{0}$ which impose some restriction on $\rho$ and $p$. In our result we remove this condition, so Theorem 2.3 is weaker then Theorem 2 in [10].

Remark 2.2. To give similar results for the equation

$$
\left(r(t) \psi(u)\left|u^{\prime}\right|^{\alpha-1} u^{\prime}\right)^{\prime}+Q(t, u)=P\left(t, u, u^{\prime}\right)
$$

where $r \in C\left(I, R^{+}\right), \quad \psi \in C\left(R, R^{+}\right), R=(-\infty, \infty)$, $I=\left[t_{0}, \infty\right)$ and $\alpha$ is a positive real number, still remains as an open problem and will be interesting.

\section{Example}

Consider the differential equation of the form

$$
\left(e^{4 t} u^{2} u^{\prime}\right)^{\prime}+\left(e^{6 t}+\theta_{1}(t, u)\right) u^{5}=\theta_{2}\left(t, u, u^{\prime}\right) u^{5}, t \geq t_{0}
$$

where $\theta_{1}$ and $\theta_{2}$ are continuous function such that $\theta_{1}(t, u) \geq 0$ and $\theta_{1}\left(t, u, u^{\prime}\right) \leq 1$. All conditions of Theorem 2.1 are satisfied. Then every nonoscillatory solutions $u$ of (16) we have $\lim _{t \rightarrow \infty} \inf |u(t)|=0$. In particular

$$
\left(e^{4 t} u^{2} u^{\prime}\right)^{\prime}+\left(e^{6 t}+e^{-2 t}\right) u^{5}=e^{-2 t} u^{5}, t \geq 0
$$

has a solution $u(t)=e^{-t}$ and $\liminf _{t \rightarrow \infty}|u(t)|=0$.

\section{REFERENCES}

[1] R. P. Agarwal, S. R. Grace and D. O. Regan, "Oscillation Theory for Second Order Linear, Half Linear, Superlinear, Sublinear Dynamic Equations,” Kluwer, Dordecht, 2002. doi:10.1016/0022-247X(91)90185-3

[2] S. R. Grace, "Oscillatory and Asymptotic Behavior of Certain Functional Differential Equations," Journal of Mathematical Analysis and Applications, Vol. 62, No. 1, 1991, pp. 177-188.

[3] M. Remili, “Oscillation Criteria for Second Order Nonlinear Perturbed Differential Equations,” Electronic Journal of Qualitative Theory of Differential Equations, Vol. 25, 2010, pp. 1-11.

[4] P. Temtek and A. Tiryaki, "Oscillation Criteria for a Certain Second Order Nonlinear Perturbed Differential Equations," Submitted for Publication, 2012.

[5] Q. Zhang and L. Wang, "Oscillatory Behavior of Solutions for a Class of Second Order Nonlinear Differential Equation with Perturbation,” Acta Applicandae Mathematicae, Vol. 110, No. 2, 2010, pp. 885-893. doi:10.1007/s10440-009-9483-8

[6] M. E. Hammet, "Nonoscillation Properties of a Nonlinear Differential Equation," Proceedings of the American Mathematical Society, Vol. 30, No. 1, 1971, pp. 92-96. doi:10.1090/S0002-9939-1971-0279384-5

[7] R. P. Agarwal, S. R. Grace and D. O. Regan, "Oscillation Theory for Second Order Dynamic Equations,” Taylor and Francis, London, 2003. doi:10.4324/9780203222898

[8] Ch. G. Philos, "On the Existence of Nonoscillatory Solutions Tending to Zero at $\infty$ for Differential Equations with Positive Delays," Archiv der Mathematik, Vol. 36, No. 1, 1981, pp. 168-170. doi:10.1007/BF01223686

[9] P. J. Y. Wong and R. P. Agarwal, "Oscillation Theorems and Existence Criteria of a Asymptotically Monotone Solution for Second Order Differential Equations," Dynamic Systems \& Applications, Vol. 4, 1995, pp. 477-496.

[10] S. R. Grace and B. S. Lalli, "Oscillations in Second Order Differential Equations with Alternating Coefficients," Periodica Mathematica Hungarica, Vol. 1, 1998, pp. 69-78. 